
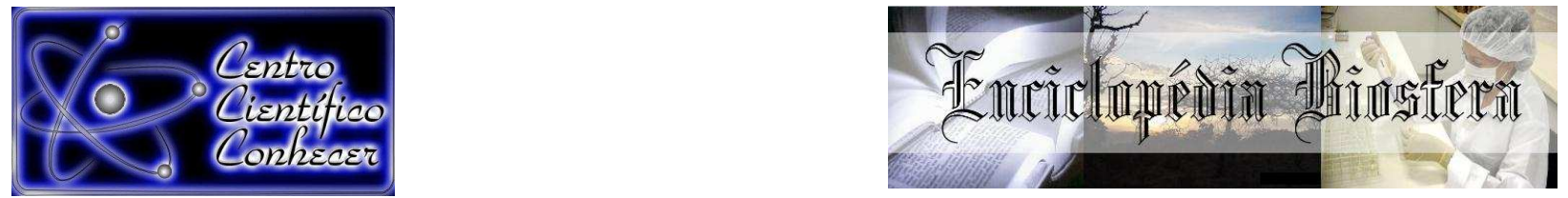

\title{
ANÁLISE TEMPORAL E ESTATÍSTICA DA VARIAÇÃO DO PREÇO DO ÁLCOOL COMBUSTÍVEL NAS PRINCIPAIS REGIÕES PRODUTORAS DO BRASIL
}

\author{
Paula Beatricy Weba Moreira ${ }^{1}$, Edilailsa Januário de Melo $^{2}$, José Izaquiel Santos da \\ Silva ${ }^{3}$
}

${ }^{1}$ Especialista em Engenharia de Campo - Qualidade, Programa de Pós-Graduação em Energia e Ambiente, Universidade Federal do Maranhão, São Luís - MA, Brasil, (paula-wm@hotmail.com)

${ }^{2}$ Mestranda do Programa de Pós-Graduação em Biocombustíveis,

Universidade Federal dos Vales do Jequitinhonha e Mucuri (UFVJM), Diamantina MG, Brasil

${ }^{3}$ Professor do Instituto de Ciência e Tecnologia da UFVJM, Diamantina - MG, Brasil

Recebido em: 03/10/2016 - Aprovado em: 21/11/2016 - Publicado em: 05/12/2016

DOI: 10.18677/EnciBio_2016B_162

\begin{abstract}
RESUMO
O mundo se tornou dependente da energia fóssil, porém existem gargalos a serem vencidos. Alternativas foram encontradas para diminuir o consumo de combustíveis fósseis, o caso dos biocombustíveis. No Brasil, a cana de açúcar é a principal matéria-prima para a produção de etanol, que pode ser produzido como álcool anidro, utilizado na mistura da gasolina, ou na forma de álcool hidratado (utilizado em carros Flex). A produção de álcool no Brasil está concentrada em duas regiões: Centro-Sul, representada por São Paulo; Norte-Nordeste, representada por Alagoas e Pernambuco. Entre essas regiões, e ao longo do tempo, existe uma variação no preço do álcool combustível. Este trabalho teve como objetivo analisar estas variações ao longo dos anos 2003-2012 e encontrar modelos estatísticos que expliquem estas variações. O método utilizado foi o de efeitos mistos (leva em consideração efeitos aleatórios), já que a independência das variáveis explicativas não pode ser garantida. A estimação dos parâmetros foi feita pelos métodos da Máxima Verossimilhança e Máxima Verossimilhança Restrita, e a escolha do modelo foi baseada nos critérios AIC (Critério de Informação Akaike) e BIC (Critério de Informação Bayesiano). Os resultados mostraram evolução temporal do preço do álcool combustível e que os Estados de Alagoas e Pernambuco apresentaram valores aproximados no período; São Paulo, no mesmo tempo, possui os menores preços. Observou-se, com o Coeficiente de Pearson, que as variáveis explicativas têm correlação forte com a variável dependente e o modelo 3 se mostrou o melhor a partir da comparação do seu AIC e BIC.
\end{abstract}

PALAVRAS-CHAVE: AIC, Biocombustível, Estimação de Parâmetros. 


\title{
TEMPORAL AND STATISTICAL ANALYSIS OF THE VARIATION OF THE FUEL ALCOHOL PRICE IN THE MAIN PRODUCING REGIONS OF BRAZIL
}

\begin{abstract}
The world has become dependent on fossil energy, but there are bottlenecks to be overcome. Alternatives were found to reduce the consumption of fossil fuels, the case of biofuels. In Brazil, sugar cane is the main raw material for the production of ethanol, which can be produced as anhydrous alcohol, used in the gasoline blend, or in the form of hydrated alcohol (used in Flex cars). Alcohol production in Brazil is concentrated in two regions: Center-South, represented by Sao Paulo; NorthNortheast, represented by Alagoas and Pernambuco. Between these regions, and over time, there is a variation in the price of fuel alcohol. This study aimed to analyze these variations over the years 2003-2012 and to find statistical models that explain these variations. The method used was that of mixed effects (taking into account the random effects), since the independence of explanatory variables cannot be guaranteed. The parameters were estimated using the Maximum Likelihood and Maximum Restricted Likelihood methods, and the model choice was based on the Akaike Information Criteria (AIC) and BIC (Bayesian Information Criteria) criteria. The results showed time evolution of the fuel alcohol price and that the states of Alagoas and Pernambuco presented approximate values in the period; Sao Paulo, at the same time, has the lowest prices. The Pearson Coefficient showed that the explanatory variables have a strong correlation with the dependent variable and the model 3 showed the best from the comparison of its AIC and BIC.
\end{abstract}

KEYWORDS: Parameter Estimation, Biofuel, AIC.

\section{INTRODUÇÃO}

O balanço energético é um termo utilizado para indicar a relação entre a energia. Os biocombustíveis se disseminaram no Brasil e no mundo como fonte energética alternativa. $O$ etanol, feito a partir de oleaginosas como o milho e a cana de açúcar, é uma fonte de energia renovável, e com isso, segundo a Fundação Getúlio Vargas (FGV PROJETOS, 2013), esta fonte tem a oferta definida essencialmente por fatores exógenos como: tecnologia de produção, características físico-climáticas da região produtora, custos de insumos, mão de obra e outras despesas operacionais, além de políticas agrícolas e energéticas.

Nos Estados Unidos e no mundo, os fatores que mais influenciam na variação do preço do etanol são o preço do petróleo, pois os derivados são amplamente utilizados em atividades agroindustriais, e as políticas de subsídios ao etanol combustível. No Brasil, porém, este cenário se difere, isto se deve a uma característica das usinas brasileiras de produzirem etanol ou açúcar, ou seja, estas usinas têm a capacidade de direcionar a produção de acordo com os preços do mercado (ERNST \& YOUNG TERCO, 2012).

Outra característica brasileira acerca do mercado do etanol que o diferencia em relação ao resto do mundo: a adoção dos motores flex, que funcionam tanto com gasolina como com álcool hidratado, sendo do consumidor a decisão pelo combustível levando em consideração não apenas o preço, mas também fatores como diferenciais de custo de manutenção, entre outros (ANP, 2013). 
Porém, esta possibilidade de escolha tem menos impacto à medida que a gasolina possui uma proporção de etanol em sua composição de $20-25 \%$. Então um aumento da participação da gasolina aumenta, em menor escala, o consumo do álcool anidro, que é um etanol mais concentrado, produzido especialmente para esta mistura e consequentemente diminui a demanda do álcool hidratado feito a partir do anidro que é usado nos motores flex (ERNST \& YOUNG TERCO, 2012).

No Brasil, a produção do álcool hidratado e anidro esta concentrada em duas regiões, a Centro-Sul representada pelo Estado de São Paulo, e a NorteNordeste representada pelos Estados de Alagoas e Pernambuco. Segundo SIGNORINI \& MARJOTTA-MAISTRO (2007), os preços do álcool hidratado e anidro sofrem variações semelhantes. Estas variações começam no Estado de São Paulo, que concentra $90 \%$ da produção do país, e esta tendência é acompanhada pelos outros Estados produtores.

Este mesmo estudo demonstrou que apesar da semelhança na variação de preços das regiões produtoras, estes são maiores na região Nordeste. Este fato é causado principalmente pelo custo de transporte entre as regiões. Verificou-se também uma maior correlação entre os preços nos Estados de Alagoas e Pernambuco, em função da proximidade (SIGNORINI \& MARJOTTA-MAISTRO, 2007).

Conforme apresentado por MALHOTRA (2001), um modelo estatístico é um conjunto de variáveis e seus interrelacionamentos, concebido para representar de maneira completa ou parcial um sistema ou um processo real. Diferentes modelos estatísticos são apresentados na literatura (FERNANDES, 1999), os modelos de regressão são largamente utilizados em diversos campos da ciência com intuito de prever, identificar e explicar fenômenos, dentre os modelos mais comumente utilizados estão os lineares e não lineares múltiplos (COSTA, 2010).

O principal objetivo dos modelos de regressão é explicar, o comportamento de uma variável $Y$, dita variável resposta, em função de outras variáveis $X_{1}, \ldots, X_{\mathrm{p}}$, chamadas de preditores, variáveis explicativas ou variáveis preditivas. Para isso, parte-se sistematicamente de um conjunto de dados que consiste em um determinado número, $n$, de realizações do vetor $\left(Y, X_{1}, \ldots, X_{p}\right)$ (FURLAN, 2009).

Os modelos de efeitos mistos são utilizados para descrever a relação entre uma variável resposta e uma ou mais covariáveis em dados agrupados de acordo com um ou mais fatores (COSTA, 2010).

Neste contexto, o presente trabalho teve por objetivo analisar as variações nos preços do álcool anidro entre os principais Estados produtores: São Paulo, Alagoas e Pernambuco em um período de 10 anos (2003-2012), como também identificar os fatores que influenciam a variação dos preços, utilizando o método estatístico dos efeitos mistos.

\section{Base de Dados}

\section{MATERIAL E MÉTODOS}

Os dados utilizados neste estudo se referem ao preço do álcool anidro no período de 2003 a 2012 em três estados com maior produção de AAC (Álcool Anidro Combustível) no Brasil, representados por Pernambuco, Alagoas e São Paulo. O Estado de São Paulo concentra $60 \%$ da produção nacional. Estes três estados demonstram condições climáticas e solos favoráveis para o cultivo da cana de açúcar, a matéria-prima para produção do álcool fabricado no Brasil. 
Neste contexto, os estados de São Paulo, Alagoas e Pernambuco foram escolhidos como representantes para efeito deste estudo, que visa determinar a evolução temporal dos preços do álcool no Brasil no período de 10 anos e quais fatores são significativos para esta variação.

\section{Coleta dos dados}

Os dados obtidos para este estudo são secundários, advindos de fontes mostradas no Quadro 1. Dentre os dados, estão as variáveis explicativas ou independentes e a variável resposta ou dependente e ainda as variáveis intervenientes, representadas pelos estados. Estas foram levantadas mensalmente no período entre 2002 a 2013.

QUADRO 1- Variáveis e suas respectivas fontes.

\begin{tabular}{c|c|c}
\hline VARIÁVEL & FONTE & SITE \\
\hline $\begin{array}{c}\text { Preços do Açúcar Cristal e } \\
\text { do Álcool Anidro } \\
\text { Combustível }\end{array}$ & $\begin{array}{c}\text { Centro de Estudos } \\
\text { Avançados em Economia } \\
\text { - CEPEA - (2013) }\end{array}$ & www.cepea.esalq.usp.br \\
\hline $\begin{array}{c}\text { Preços do contrato futuro } \\
\text { de açúcar bruto no11 da } \\
\text { Csce/Nybot }\end{array}$ & $\begin{array}{c}\text { Bolsa de futuro New York } \\
\text { Board of Trade (Nybot) } \\
(2013)\end{array}$ & www.nybot.com \\
\hline Taxa de câmbio comercial & $\begin{array}{c}\text { Instituto de Pesquisa } \\
\text { Econômica Aplicada } \\
\text { (IPEA) (2014) }\end{array}$ & www.ipeadata.gov.br \\
\hline $\begin{array}{c}\text { Bnited States } \\
\text { e Europe Brent Spot }\end{array}$ & $\begin{array}{c}\text { Uepartament of Agriculture } \\
\text { (USDA) (2014) }\end{array}$ & www.usda.gov \\
\hline
\end{tabular}

Fonte: Elaborado pelos autores.

\section{Variáveis em Estudo}

As variáveis explicativas utilizadas neste trabalho foram o açúcar (o Brasil desponta com $40 \%$ das exportações mundiais, e este é originado da mesma matéria-prima do álcool) e a cana de açúcar (alguns estudos consideraram o potencial dessa commodity sobre a formação do preço do álcool). Com isso, foram escolhidas as variáveis: açúcar cristal e taxa de câmbio comercial (PENEDO et al., 2008).

Como o álcool anidro é utilizado na mistura da gasolina, os preços destes produtos podem ter relação entre si, e como o álcool é feito a partir da cana de açúcar e a gasolina a partir do petróleo, foram escolhidas as variáveis: preços do barril de petróleo WTI e barril de petróleo Brent, que se tratam do petróleo negociado nas bolsas de Nova York e Londres, respectivamente. As séries do barril de petróleo, WTI Spot e Europe Brent Spot foram obtidas junto ao United States Departament of Agriculture (USDA).

O álcool hidratado também foi escolhido como variável explicativa, pois pode ser produzida a partir do álcool anidro, então acredita-se que existe influência entre os preços dos mesmos. Para obtenção das variáveis acima citadas, diferentes fontes foram utilizadas. As séries de preços do Açúcar Cristal que é dado em sacas de $50 \mathrm{~kg}$ e do Álcool Anidro Combustível e do Álcool hidratado dados em US\$/litro, 
são aferidas pelo CEPEA - Centro de Estudos Avançados em Economia Aplicada da Escola Superior de Agricultura "Luiz de Queiroz" da Universidade de São Paulo. Já a série da taxa de câmbio comercial em (R\$/US\$) foi obtida junto ao Instituto de Pesquisa Econômica Aplicada (IPEA).

As variáveis intervenientes existentes nesta análise são os Estados de São Paulo, representado por 1; Alagoas, que seria a variável 2, e Pernambuco sendo representada por 3 . Estes dados foram levantados com valores mensais e o período de estudo foi de 10 anos entre os anos 2003 e 2012. Estas variáveis encontram-se resumidas no Quadro 2.

QUADRO 2 - Variáveis utilizadas neste estudo.

\begin{tabular}{l|l|l}
\hline $\begin{array}{c}\text { VARIÁVEIS } \\
\text { INDEPENDENTES }\end{array}$ & $\begin{array}{c}\text { VARIÁVEIS } \\
\text { INTERVENIENTES }\end{array}$ & VARIÁVEL RESPOSTA \\
\hline$>$ Preço do açúcar; & $>$ São Paulo; & $>$ Preço do álcool \\
$>$ Cotação do dólar; & $>$ alagoas; & \\
$>$ Preço do barril de & $>$ Pernombustível. \\
petróleo WTI; & $>$ Pernambuco. & \\
$>\begin{array}{l}\text { Preço do barril de } \\
\text { petróleo Brent; }\end{array}$ & & \\
$>\begin{array}{l}\text { Preço do álcool } \\
\text { hidratado. }\end{array}$ & & \\
\hline
\end{tabular}

Fonte: Elaborado pelos autores.

\section{Análise Estatística}

\section{Análise descritiva}

Primeiramente foi realizada a análise descritiva dos dados, sendo obtidas as médias anuais do preço do AAC para cada estado e o respectivo desvio padrão. Para verificar a suposição de normalidade das variáveis em estudo foi aplicado o teste Shapiro Wilk, com nível de significância de 5\%. Para as variáveis que não apresentaram distribuição normal, foi utilizada a transformação logarítmica (SENRA et al., 2007; PENEDO et al., 2008).

Diagramas de dispersão foram construídos, considerando a variável AAC e cada uma das variáveis explicativas em estudo, com a finalidade de visualizar correlação entre tais variáveis. Também foi calculado o coeficiente de correlação de Pearson (CP). Consideraram-se como estatisticamente significantes, os valores de $p$ menores que 0,05 .

\section{Definição do modelo}

Para encontrar a variação do preço do AAC nos Estados de São Paulo, Alagoas e Pernambuco durante o período em análise, foram ajustados os modelos de efeitos mistos.

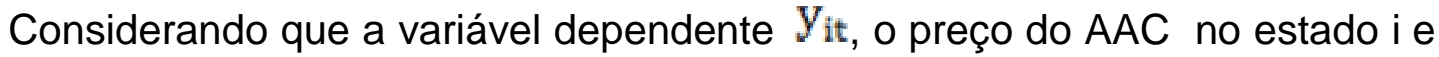
no tempo $t$, sendo $i=1,2,3$ e $t=1, . ., 10$. No Modelo 1 não são considerados efeitos aleatórios (modelo de regressão linear). Já no Modelo 2, são considerados os efeitos aleatórios apenas para os estados $(i=1,2,3)$. Por fim, o Modelo 3 , os efeitos aleatórios para cada estado e para cada ano. 
Modelo 1

$Y_{i t}=\beta o+\beta_{1 i t} x_{1 i t}+\beta_{2} x_{2 i t}+\ldots \ldots \ldots .+\beta_{p} x_{p i t}+\varepsilon_{i t}$

Modelo 2

$\mathrm{Y}_{\mathrm{it}}=\beta \mathrm{o}+\beta_{1 \mathrm{it}} \mathrm{x}_{1 \mathrm{it}}+\beta_{2} \mathrm{x}_{2 \mathrm{it}}+\ldots \ldots \ldots+\beta_{\mathrm{p}} \mathrm{x}_{\mathrm{pit}}+Z \gamma_{i}+\varepsilon_{\mathrm{it}}$

Modelo 3

$\mathrm{Y}_{\mathrm{it}}=\beta \mathrm{o}+\beta_{1 \mathrm{it}} \mathrm{x}_{1 \mathrm{it}}+\beta_{2} \mathrm{x}_{2 \mathrm{it}}+\ldots \ldots \ldots+\beta_{\mathrm{p}} \mathrm{x}_{\mathrm{pit}}+Z \gamma_{i t}+\varepsilon_{\mathrm{it}}$

Sendo:

$\beta_{0}=$ Valor do intercepto dos efeitos fixos;

$\beta_{1}=$ Valor do intercepto da variável preço do açúcar;

$\beta_{2}=$ Valor do intercepto da variável taxa de câmbio;

$\beta_{3}=$ Valor do intercepto da variável cotação do petróleo tipo WTI;

$\beta_{4}=$ Valor do intercepto da variável cotação do petróleo tipo BRENT;

$\beta_{5}=$ Valor do intercepto da variável preço do álcool hidratado

$X_{1 t i}=$ Preço do açúcar no estado i e ano t;

$X_{2 t i}=$ Taxa de câmbio no estado $\mathrm{i}$ e ano $\mathrm{t}$;

$X_{3 t i}=$ Cotação petróleo tipo WTI no estado i e ano t;

$X_{4 t i}=$ Cotação petróleo tipo BRENT no estado i e ano $\mathrm{t}$;

$X_{5 t i}=$ Preço do álcool hidratado no estado i e ano t.

Utilizaram-se os métodos de Máxima Verossimilhança (ML) e Máxima Verossimilhança Restrita (REML) para estimação dos parâmetros usados para construção do modelo, e a escolha foi feita a partir do critério de AIC (Critério de informação Kaike), cujos valores foram obtidos no software livre estatístico R, usado neste trabalho. Estes critérios foram criados para permitir a comparação entre os modelos construídos. Segundo estes critérios quanto menor for o valor do critério de informação, melhor o modelo (COSTA, 2010). De acordo com PEREIRA (2009), para modelos ajustados por REML, estes critérios somente podem ser comparados entre os modelos com a mesma estrutura de efeitos fixos.

Para a escolha da covariância que melhor explique a variabilidade e a correlação entre as medidas repetidas e consequentemente escolher o modelo, utilizam-se métodos no qual se destacam o AIC (Critério de Informação Kaike) e o BIC (Critério de Informação Bayesiana). Estes são nada mais do que valores para os logaritmos das funções de verossimilhanças do modelo, que dependem do número de observações e do número de parâmetros do modelo (PEREIRA, 2009). 


\section{RESULTADOS E DISCUSSÃO}

\section{Análise descritiva}

Para analisar a variação do preço do álcool anidro durante o período de 10 anos foi construído um gráfico de linhas (Figura 1), demonstrando o comportamento anual dos Estados de São Paulo, Alagoas e Pernambuco. Pode-se observar o comportamento não linear, porém uma evolução crescente.

Pode-se observar também que a evolução do preço do álcool anidro foi de menos de US\$ 0,20 em 2004 até US\$0,98, o maior valor alcançado foi do Estado de Pernambuco em meados de 2011, porém os preços não ultrapassaram US $\$ 1,00$. Lembrando que este valor é do álcool anidro que é adicionado à gasolina em proporção de $25 \%$.

Também na Figura 1, nota-se que os estados da região norte-nordeste, Alagoas e Pernambuco apresentam valores praticamente iguais em todo o período em estudo e que o Estado de São Paulo é o que mais se diferencia dos demais, porém sempre com os menores preços. Este comportamento pode ser explicado pela proximidade dos Estados de Alagoas e Pernambuco e em contraponto a distância das mesmas do Estado de São Paulo.

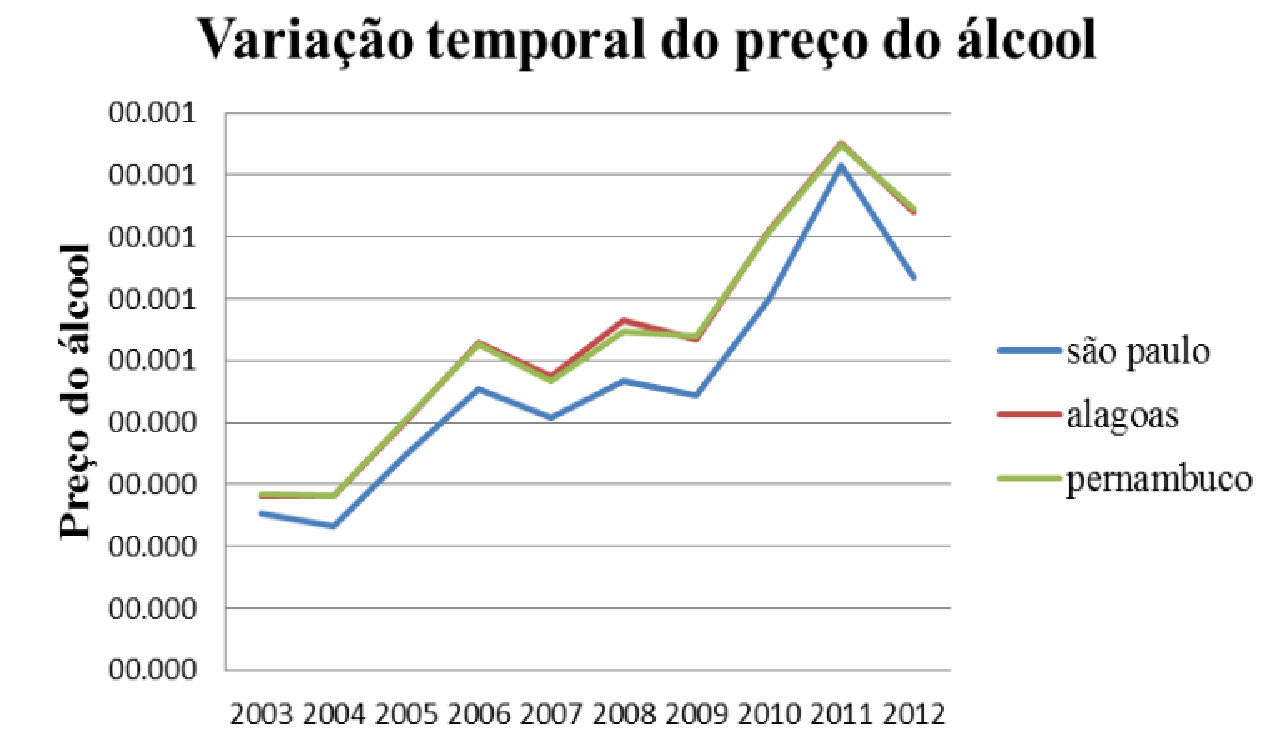

FIGURA 1 - Variação temporal do preço do álcool anidro combustível entre os anos 2003 a 2012. Fonte: Elaborado pelos autores.

Com o objetivo de verificar a correlação entre as variáveis explicativas e a variável dependente, foram feitos gráficos de dispersão, mostrados na Figura 02, do preço do álcool anidro com cada uma das variáveis explicativas e calcularam-se seus respectivos coeficientes de correlação de Pearson. Para a primeira variável X1, ou seja, preço do açúcar, observa-se um comportamento linear, e uma correlação positiva nos três Estados em estudo, confirmado pelo valor do coeficiente de Pearson que se aproxima de 1, com valores bem aproximados entre si. Para a 
variável X2, taxa de câmbio, observa-se uma correlação linear negativa. Esta negatividade se explica pelo fato do preço do álcool anidro e das demais variáveis estejam em dólares (US\$) e a taxa de câmbio é dada por R\$/US\$, ou seja, esta relação é inversamente proporcional. A maior correlação é para o Estado de Alagoas com um coeficiente de Pearson que mais se aproxima de -1 .

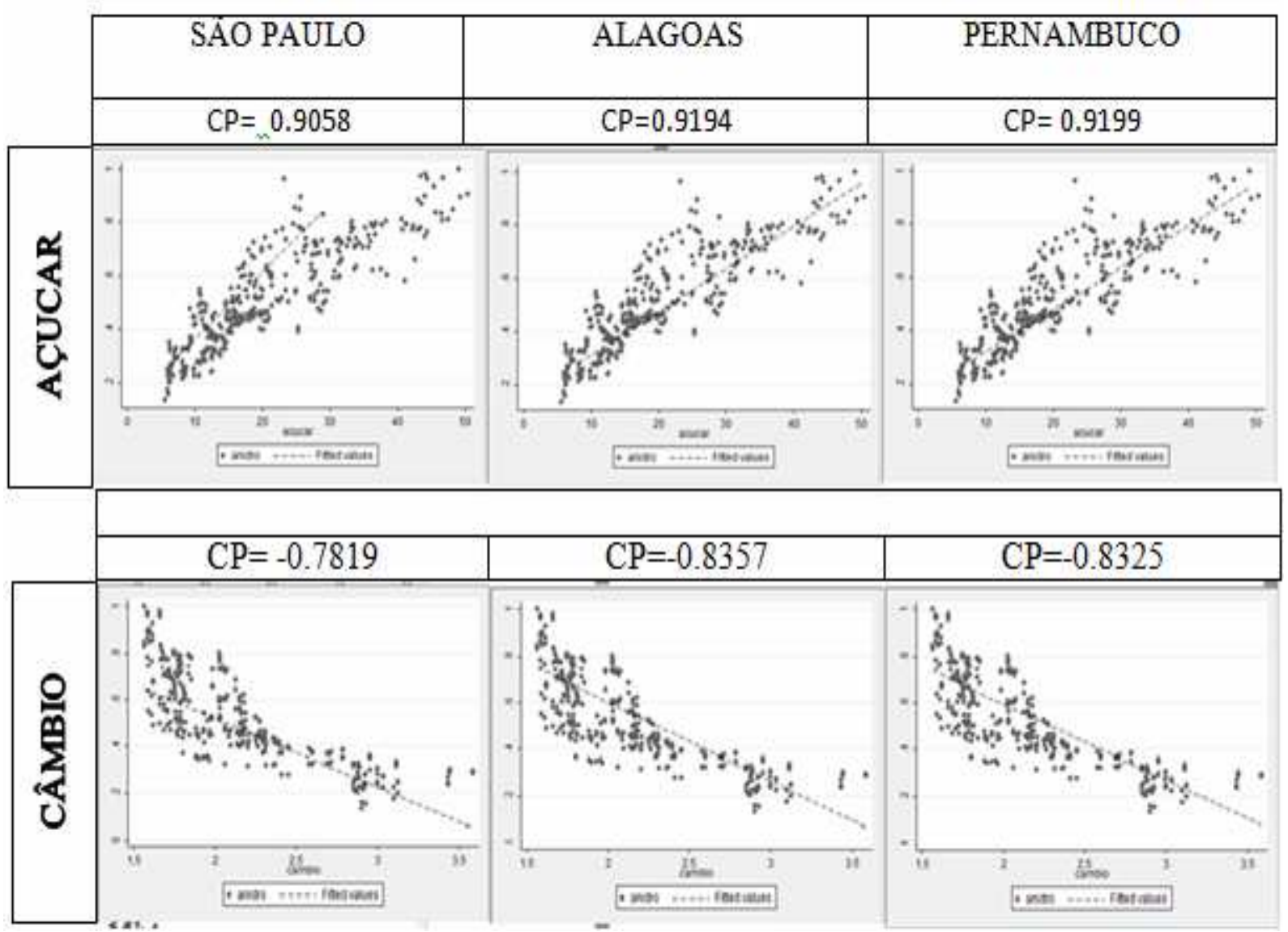

FIGURA 2 - Gráficos de dispersão e Coeficiente de correlação de Pearson (CP) para as variáveis preço do açúcar e taxa de câmbio. Fonte: Elaborado pelos autores.

Para as variáveis, cotação do barril de petróleo WTI e Brent, o comportamento (Figura 3) se mostrou linear com correlação positiva. De acordo com o Coeficiente de Pearson (CP), esta correlação pode ser considerada forte para os três Estados, explicada pelo fato de que o álcool anidro é parte da mistura da gasolina, onde o valor é determinado pelo preço de barril de petróleo negociado nas bolsas de Nova York e Londres. 


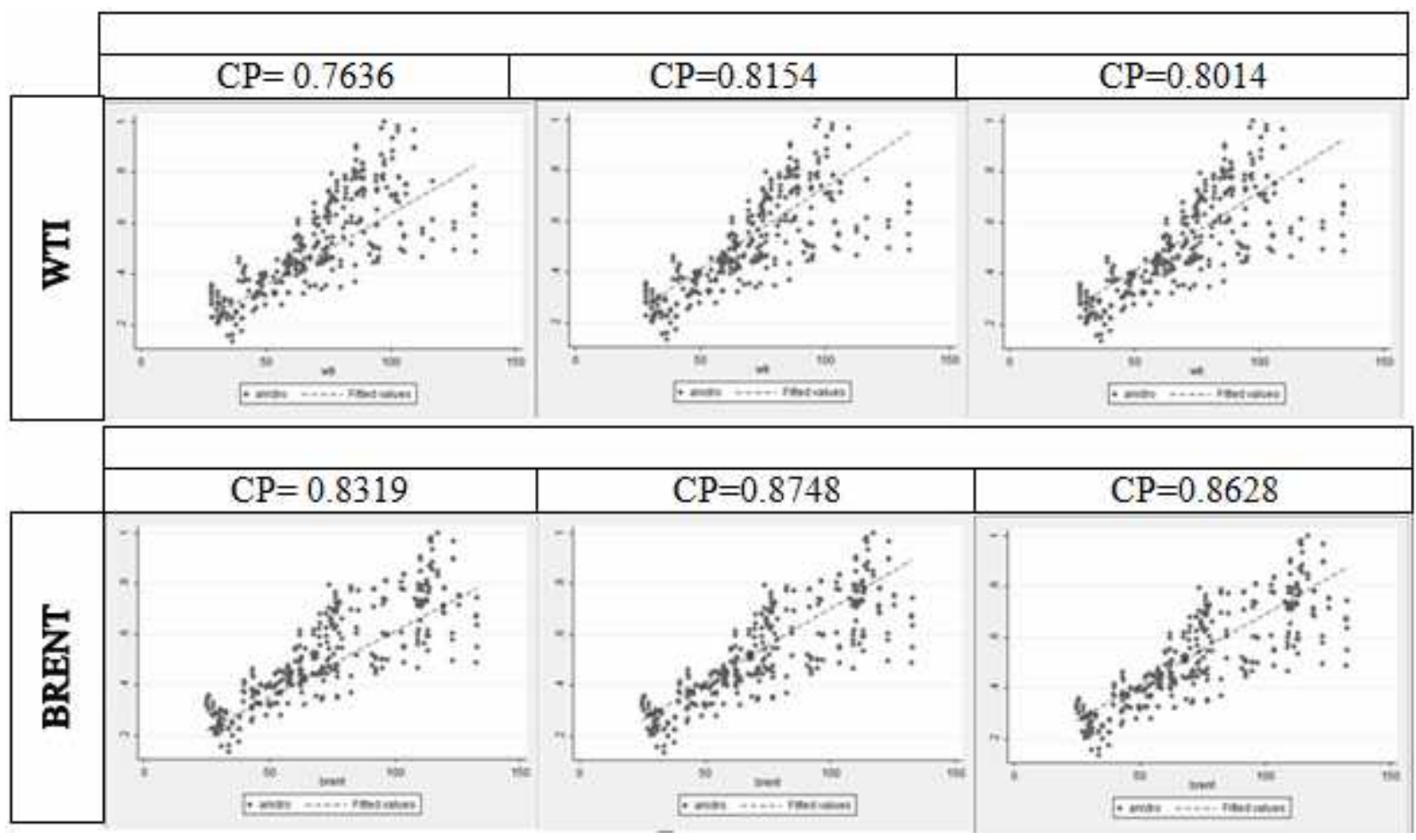

FIGURA 3 - Gráficos de Dispersão e Coeficientes de Pearson (CP) para as variáveis barril de petróleo WTI e Brent. Fonte: Elaborado pelos autores.

Para o álcool hidratado (Figura 4), esta correlação se mostrou a mais forte quando comparada às demais, chegando a valores entre 0,99 para os Estados de São Paulo e Alagoas. Esta correlação pode ser considerada quase perfeita. $O$ motivo desta correlação quase perfeita está no fato de que o álcool anidro e hidratado são produtos de uma mesma matéria-prima, a cana de açúcar, e o álcool anidro são produzidos a partir do hidratado.

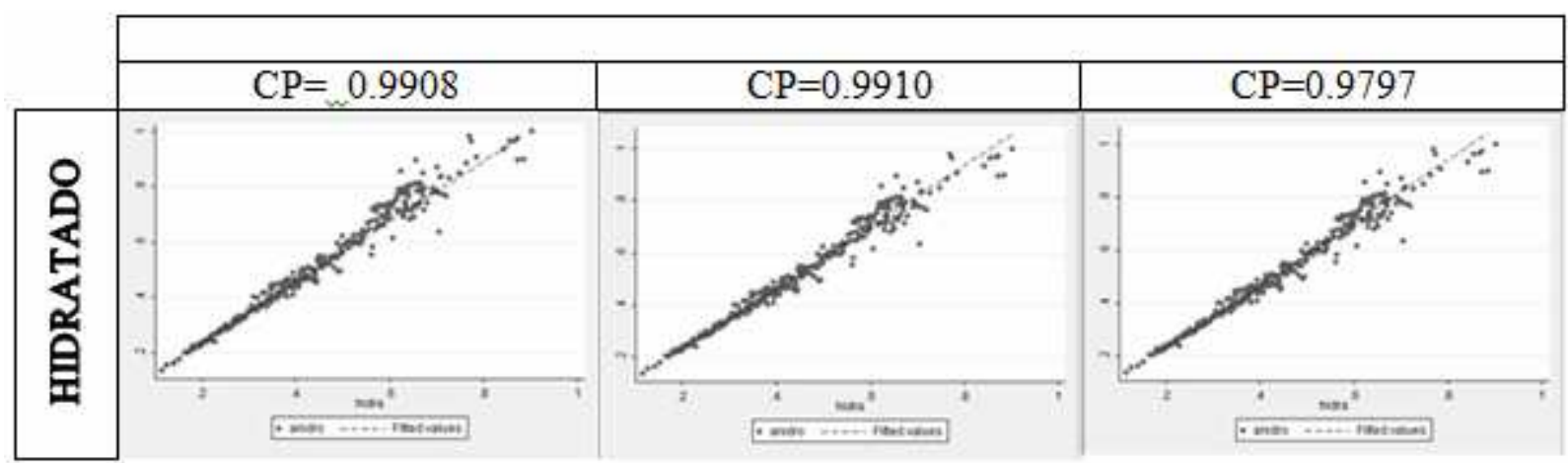

FIGURA 4 - Gráficos de Dispersão e Coeficientes de Pearson (CP) para a variável preço do álcool hidratado. Fonte: Elaborado pelos autores.

Então, as variáveis que apresentaram uma correlação mais forte com o álcool anidro, foram o álcool hidratado e o açúcar, ambos produzidos com a mesma matéria-prima do álcool anidro, a cana de açúcar, fato este que pesou consideravelmente na influência destas variáveis sobre o preço do AAC. Em relação aos estados, o que apresentou em todas as variáveis os maiores coeficientes de Pearson foi o estado de Alagoas. 
$\mathrm{Na}$ Tabela 3, são apresentados os valores dos preços médios anuais do AAC para cada Estado e seus respectivos desvios padrão.

TABELA 1 - Médias anuais do preço em dólar do álcool anidro e seus respectivos desvios padrão.

\begin{tabular}{c|c|c|c}
\hline \multicolumn{3}{c}{ ÁLCOOL ANIDRO } \\
\cline { 2 - 4 } ANO & SÃO PAULO & ALAGOAS & PERNAMBUCO \\
\hline 2003 & $0,2523( \pm 0,3866)$ & $0,2824( \pm 0,0373)$ & $0,2841( \pm 0,0438)$ \\
\hline 2004 & $0,2324( \pm 0,0728)$ & $0,2818( \pm 0,0565)$ & $0,2814( \pm 0,0570)$ \\
\hline 2005 & $0,3476( \pm 0,0574)$ & $0,4015( \pm 0,0329)$ & $0,4042( \pm 0,0309)$ \\
\hline 2006 & $0,4547( \pm 0,0557)$ & $0,5300( \pm 0,0601)$ & $0,5272( \pm 0,0746)$ \\
\hline 2007 & $0,4082( \pm 0,0596)$ & $0,4738( \pm 0,0301)$ & $0,4679( \pm 0,0316)$ \\
\hline 2008 & $0,4669( \pm 0,0538)$ & $0,5652( \pm 0,1124)$ & $0,5462( \pm 0,0850)$ \\
\hline 2009 & $0,4440( \pm 0,1267)$ & $0,5331( \pm 0,1103)$ & $0,5385( \pm 0,1061)$ \\
\hline 2010 & $0,5981( \pm 0,1017)$ & $0,7088( \pm 0,0659)$ & $0,7082( \pm 0,0744)$ \\
\hline 2011 & $0,7517( \pm 0,1995)$ & $0,8346( \pm 0,2627)$ & $0,7201( \pm 0,2796)$ \\
\hline 2012 & $0,6346( \pm 0,0522)$ & $0,7392( \pm 0,0290)$ & $0,7402( \pm 0,0285)$ \\
\hline
\end{tabular}

Fonte: Elaborado pelos autores.

Pela análise das informações da tabela 3 pode-se observar que não houve grande variação no preço do álcool anidro para os Estados de Alagoas e Pernambuco nos anos de 2003 e 2004 e que esses dois Estados têm valores semelhantes em quase todos os anos estudados, este fato pode ser explicado pela proximidade geográfica entre os dois Estados.

No Estado de São Paulo, existe uma diferença de preço em relação aos outros Estados. Esta diferença está associada principalmente a uma maior eficiência na produção. No estado de São Paulo as usinas possuem uma maior tecnologia e a cana-de-açúcar é de melhor qualidade devido as condições climáticas mais favoráveis. Este estado detém $60 \%$ da produção nacional, apresentando em todos os anos analisados os menores preços do AAC.

\section{Modelos Mistos}

Para iniciar a modelagem, foi atestada a não normalidade dos dados. Então se mostrou necessário a transformação desses dados para função logarítmica. Com isso, foram obtidos 12 modelos com estimação de parâmetros a partir dos métodos de Máxima Verossimilhança (ML) e Máxima Verossimilhança Restrita (REML) e obtidos valores de AIC e BIC, demonstrados na Tabela 2. Conforme esta tabela, 
observa-se que os valores de AIC e BIC são menores para o Modelo 3, com estimação dos parâmetros pelo método ML.

TABELA 2 - Critérios de avaliação para cada modelo, sem e com transformação.

\begin{tabular}{|c|c|c|c|c|}
\hline \multicolumn{5}{|c|}{ MODELOS SEM TRANSFORMAÇÃO } \\
\hline & \multicolumn{2}{|c|}{ AIC } & \multicolumn{2}{|c|}{$\mathrm{BIC}$} \\
\hline & REML & $\mathrm{ML}$ & REML & ML \\
\hline 1 & -1492.486 & -1565.801 & -1446.054 & -1519.167 \\
\hline 2 & -1499.989 & -1573.376 & -1469.034 & -1542.287 \\
\hline 3 & -1514.197 & -1584.357 & -1479.373 & -1549.382 \\
\hline \multicolumn{5}{|c|}{ MODELOS COM TRANSFORMAÇÃO } \\
\hline 1 & -1138.789 & -1181.713 & -1092.357 & -1135.079 \\
\hline 2 & -1177.893 & -1189.137 & -1143.069 & -1158.048 \\
\hline 3 & -1146.189 & -1217.061 & -1115.235 & -1182.086 \\
\hline
\end{tabular}

Fonte: Elaborado pelos autores.

A Tabela 2 mostra que os valores de AIC e BIC são menores para o Modelo 3, com estimação dos parâmetros pelo método ML. A Tabela 3 apresenta as estimativas dos parâmetros do Modelo 3. Apenas a variável taxa de câmbio não esteve estatisticamente associada com o preço do AAC no período em estudo $(p=0,0691)$.

TABELA 3 - Resultados para o Modelo 3.

\begin{tabular}{c|c|c|c}
\cline { 2 - 4 } & VALOR & DP & P-VALOR \\
\hline INTERCEPTO & -0.0096004 & 0.12044734 & 0.9365 \\
& & & \\
\hline LOGACUCAR & 0.0408794 & 0.01327302 & 0.0022 \\
& & & 0.0691 \\
\hline LOGCAMBIO & -0.0668423 & 0.03665014 & 0.0002 \\
\hline LOGWTI & 0.1945912 & 0.05113105 & 0.0001 \\
\hline LOGBRENT & 0.1899412 & 0.04751278 & 0.0000 \\
\hline LOGHIDRA & 0.8873217 & 0.02017087 & \\
\hline
\end{tabular}

Fonte: Elaborado pelos autores. 
Como a taxa de câmbio, baseada na metodologia escolhida não foi significante, foi proposto um novo ajuste do modelo excluindo a referida variável. Deve-se se salientar que diversos estudos evidenciam uma grande influência da taxa de câmbio no preço do etanol. O modelo final ajustado (Tabela 4) demonstra que as demais variáveis estão estatisticamente correlacionadas positivamente com o preço do álcool anidro, ou seja, um aumento em qualquer uma das variáveis acarreta em aumento no preço do álcool anidro.

TABELA 4 - Resultados do Modelo 3 ajustado.

\begin{tabular}{c|c|c|c}
\cline { 2 - 4 } & VALOR & $\mathrm{DP}$ & P-VALOR \\
\hline INTERCEPTO & -0.1583485 & 0.08892247 & 0.0759 \\
\hline LOGACUCAR & 0.0430462 & 0.01326292 & 0.0013 \\
\hline LOGWTI & 0.1756676 & 0.05023083 & 0.0005 \\
\hline LOGBRENT & 0.1943623 & 0.04760593 & 0.0001 \\
\hline LOGHIDRA & 0.8934977 & 0.01994961 & 0.0000 \\
\hline
\end{tabular}

Fonte: Elaborado pelos autores.

Com o Modelo 3 ajustado, verificou-se que as hipóteses de que o preço do açúcar, do barril de petróleo WTI e Brent e do álcool hidratado são significativos para explicar a variação do preço do álcool anidro combustível. O intercepto associado aos efeitos fixos tem valor negativo.

Estes resultados mostraram que o açúcar tem relevância para explicar a variação do preço do álcool anidro, isso ocorre porque estes são produtos relacionados, tem a mesma matéria-prima, a cana de açúcar. E, como as usinas brasileiras são do tipo misto, uma variação no preço do açúcar reflete no preço do álcool. Segundo TOKGOZ \& ELOBEID (2006), o preço das duas commodities que direcionam a produção das usinas para o produto de maior valor no mercado internacional. BACCHI (2005) afirma que a produção de açúcar tem maior influência no mercado do etanol, o autor menciona que as variações no preço do açúcar no mercado doméstico impactam diretamente no preço do etanol.

As variáveis: cotação do barril de petróleo WTI e Brent também demonstraram significância que pode ser explicada pelo fato de que a gasolina, produto do petróleo, "concorre" com o álcool no Brasil por possuir a tecnologia flex. Então quando a cotação do petróleo aumenta, o preço da gasolina também tende a subir, influenciando para um crescimento no preço do álcool. BOFF (2011) aborda as principais características da indústria do etanol, segundo o autor além da influência do açúcar na produção do etanol, a demanda por gasolina também influencia, uma vez que este combustível possui $1 / 4$ de álcool anidro em sua composição. 
Alguns estudos também demonstraram a relação dessas variáveis com a variação do preço do AAC, como em PENEDO et al. (2008), que, utilizando-se de um modelo de regressão linear múltipla, encontraram resultados semelhantes aos obtidos neste trabalho. Na pesquisa de Penedo e colaboradores foram aceitas as hipóteses de que a cotação do barril de petróleo WTI, a taxa de câmbio e o preço do açúcar contribuem diretamente na variação do preço do $A A C$, ou seja, um incremento em qualquer uma dessas variáveis causa um aumento proporcional na variável dependente.

\section{CONCLUSÕES}

Com este trabalho, foi possível verificar que a variação do preço do álcool anidro entre 2003 e 2012 nos Estados de São Paulo, Alagoas e Pernambuco se comportou de maneira não linear, porém com uma evolução crescente. Além de mostrar que os Estados de Alagoas e Pernambuco se assemelham em relação ao preço do álcool, atribuído a proximidade, e o Estado de São Paulo apresentou, em todos os anos, o menor preço, explicado pela detenção de $60 \%$ da produção de álcool naquele estado.

Os diagramas de dispersão confirmaram a correlação entre as variáveis explicativas e a variável dependente, porém a variável câmbio se mostrou negativa, explicada pela relação inversamente proporcional da taxa de cambio (R\$/US\$) com o dólar, moeda adotada neste estudo. Estes diagramas demonstraram também que correlação mais forte entre as variáveis em estudo foi em relação ao preço do álcool hidratado seguido do preço do açúcar que possuem a mesma matéria prima do AAC, a cana de açúcar, sendo este fato determinante para a influência destas variáveis no preço do AAC. Em relação aos estados, Alagoas apresentou os menores valores para o Coeficiente de Pearson em todas as variáveis.

O modelo que apresentou os menores critérios de informação, foi o Modelo 3, com estimação de parâmetros por ML. Neste modelo, a variação ocorre entre os Estados e o tempo (2003-2012). A variável taxa de câmbio se mostrou não significante, esta foi retirada e o modelo ajustado mostrou que as variáveis explicam a variação do álcool anidro, ou seja, um incremento no valor de qualquer uma das variáveis aumenta o preço do AAC, destacando as variáveis: preço do álcool hidratado e açúcar que apresentaram os maiores valores. Estes bons resultados evidenciam a eficácia do método do modelo de efeitos mistos que levam em consideração os efeitos aleatórios.

\section{AGRADECIMENTOS}

Os autores agradecem à UFMA e ao Programa PROMIMP da Petrobras pelos incentivos e apoio. Também agradecem ao ICT/UFVJM.

\section{REFERÊNCIAS}

ANP - AGÊNCIA NACIONAL DO PETRÓLEO, GÁS NATURAL E BIOCOMBUSTÍVEIS. (2013). < http://www.anp.gov.br/>.

BACCHI, M. R. P. (2005). Formação de preços no setor sucroalcooleiro da Região Centro-Sul do Brasil: Relação com o mercado de combustível fóssil. In XXXIII Encontro Nacional da ANPEC, 6 a 9 de dezembro de 2005, Natal, RN. Disponível em: http://www.anpec.org.br/encontro2005/artigos/A05A143 
BOFF, H. P. (2011). Modeling the Brazilian ethanol market: How flex-fuel vehicles are shaping the long run equilibrium. China-USA Business Review, 10(4), 245-264. Bolsa de futuro New York Board of Trade (Nybot). Disponível em <www.nybot.com>

BOLSA DE FUTURO NEW YORK BOARD OF TRADE (Nybot). (2013). Disponível em <www.nybot.com>

CENTRO DE ESTUDOS AVANÇADOS EM ECONOMIA APLICADA (CEPEA/USP). (2013). Indicador dos preços de açúcar e álcool. Disponível em: http://www.cepea.esalq.usp.br.

COSTA, T. R. Modelos lineares mistos: uma aplicação na produção de leite de vacas da raça Sindi. 79f Dissertação de Mestrado. Universidade Federal Rural de Pernambuco. Pernambuco, 2010;

ERNST \& YOUNG TERCO. (2012). Brasil sustentável - Perspectivas dos mercados de petróleo, etanol e gás. < http://fgvprojetos.fgv.br/sites/fgvprojetos.fgv.br/files/estudo_20.pdf >.

FERNANDES, E. M. G. P. Estatística Aplicada. Universidade do Minho, Braga, 1999;

FGV PROJETOS. (2013). <www.portal.fgv.br>.

FURLAN, B. B. Modelagem estatística aplicada à valorização de ações. $124 \mathrm{f}$ Monografia (Bacharelado em Engenharia de Produção). Universidade de São Paulo. São Paulo, 2009;

INSTITUTO DE PESQUISA ECONÔMICA APLICADA (IPEA). (2014). Taxa de câmbio comercial. Disponível em: <http://www.ipeadata.gov.br>.

MALHOTRA, N. K. Pesquisa de Marketing. Porto Alegre: Bookman, 2001;

PENEDO, A. S. T.; PACAGNELLA, A. C. Jr.; OLIVEIRA, M. M. B. (2008). Análise Estatística Multivariada da Variação do Preço do Álcool Combustível no Estado de São Paulo. Revista Eletrônica de Administração - FACEF. v.11, 12 a ed. JaneiroJulho 2008. < http://periodicos.unifacef.com.br/index.php/rea/article/view/209 >

PEREIRA, L. M. Modelo de formação de preços de commodities agrícolas aplicado ao mercado de açúcar e álcool. 209f Tese de Doutorado. Universidade de São Paulo. São Paulo, 2009;

SENRA, L. F. A. C.; NANCI, L. C.; MELLO, J. C. C. B. S.; MEZA, L. A. (2007). Estudo sobre métodos de seleção de variáveis em DEA. Pesqui. Oper. v.27 n‥2 Rio de Janeiro Mai/Ago. 2007. http://dx.doi.org/10.1590/S0101-74382007000200001 
SIGNORINI, G.; MARJOTTA-MAISTRO, M. C. (2007). Análises de preços de combustíveis nas regiões sudeste e nordeste. Artigo publicado pág. 37. Revista

Agroanalysis. $\quad$ Edição: $\quad$ Fevereiro, $2007 . \quad<$ http://www.cepea.esalq.usp.br/pdf/Cepea_Alcool3.pdf >

TOKGOZ, S.; ELOBEID, A. (2006). An analysis of the link between ethanol, energy, and crop markets. lowa State University Working Paper 06-WP 435. 2006.

UNITED STATES DEPARTAMENT OF AGRICULTURE (USDA). (2014). Disponível em <www.usda.gov> 